\title{
Downstream Self-Destruction of Storm Tracks
}

\author{
YOHAI KASPI AND TAPIO SCHNEIDER \\ California Institute of Technology, Pasadena, California
}

(Manuscript received 18 November 2010, in final form 18 February 2011)

\begin{abstract}
The Northern Hemisphere storm tracks have maximum intensity over the Pacific and Atlantic basins; their intensity is reduced over the continents downstream. Here, simulations with an idealized aquaplanet general circulation model are used to demonstrate that even without continents, storm tracks have a self-determined longitudinal length scale. Their length is controlled primarily by the planetary rotation rate and is similar to that of Earth's storm tracks for Earth's rotation rate. Downstream, storm tracks self-destruct: the downstream eddy kinetic energy is lower than it would be without the zonal asymmetries that cause localized storm tracks. Likely involved in the downstream self-destruction of storm tracks are the energy fluxes associated with them. The zonal asymmetries that cause localized storm tracks enhance the energy transport through the generation of stationary eddies, and this leads to a reduced baroclinicity that persists far downstream of the eddy kinetic energy maxima.
\end{abstract}

\section{Introduction}

The baroclinic eddies that dominate atmospheric variability and shape the climate in the extratropics are concentrated in storm tracks-regions of maximum transient streamfunction variance or eddy kinetic energy (EKE) (Blackmon 1976; Blackmon et al. 1977; Lau 1978). In the Northern Hemisphere (NH), there are two primary storm tracks, over the Atlantic and over the Pacific (Fig. 1b). It is well established that the formation of storm tracks and the locally enhanced EKE are a result of the strong meridional temperature gradients (baroclinicity) and diabatic heating near the eastern coasts of the major continents (Hoskins and Valdes 1990; Chang et al. 2002). But it is less clear what controls where the storm tracks terminate downstream.

Storm track eddies continue to develop downstream of their generation region (Simmons and Hoskins 1979), and they can form coherent wave packets (Lee and Held 1993), within which eddy energy is passed downstream from eddy to eddy (Orlanski and Katzfey 1991; Chang and Orlanski 1993; Chang 1993; Orlanski 1998). This downstream development of eddies appears to be responsible for the fact that the EKE maximum extends downstream of the region of maximum baroclinicity

Corresponding author address: Yohai Kaspi, California Institute of Technology 1200 E. California Blvd., Pasadena, CA 91125. E-mail: yohai@alum.mit.edu near the coasts. Chang and Orlanski (1993) suggested that as long as there is enough baroclinicity to balance dissipation and barotropic decay (Simmons and Hoskins 1980), downstream development implies that there is no intrinsic limit to the energy propagation and thus storm track length. Analyses of the kinetic energy balance of storm tracks (Lau 1978, 1979) and numerical studies (e.g., Frisius et al. 1998) show that barotropic decay can lead to the termination of storm tracks. Alternatively, it has been suggested that continents with their enhanced surface and orographic drag may reduce the storm track EKE (Chang and Orlanski 1993; Mak and Deng 2007). General circulation model (GCM) simulations with continents but without orography exhibit overall weaker but longitudinally less localized storm tracks (Broccoli and Manabe 1992). This suggests that orographic stationary waves may play a role in the maintenance and termination of storm tracks (Chang et al. 2002), possibly by modifying the barotropic background flow (e.g., Son et al. 2009), which can lead to localization of storm tracks (Lee 1995; Swanson et al. 1997).

Using simulations with an idealized GCM, we show that a longitudinally localized region of increased baroclinicity leads not only to a localized storm track that has an EKE maximum and has a well-defined length, but also to EKE depression farther downstream. Indeed, sufficiently far downstream, the EKE is lower than it would be without the local enhancement of baroclinicity. That is, storm tracks self-destruct downstream. We show 

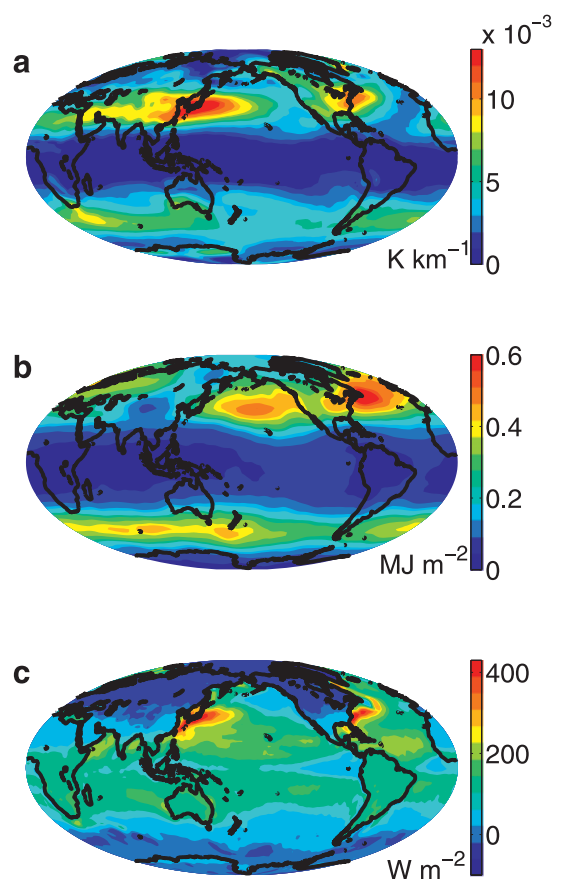

FIG. 1. NCEP reanalysis fields averaged for December-February (1970-2009). (a) Absolute value of meridional temperature gradient averaged between $300 \mathrm{hPa}$ and the surface. (b) Vertically integrated EKE, calculated using the 3-10-day bandpass-filtered zonal and meridional velocities $u^{\prime}$ and $v^{\prime}$ and defining $\mathrm{EKE}=$ $\int\left(u^{\prime 2}+v^{\prime 2}\right) d p / 2 g$. (c) Combined surface sensible and latent heat fluxes.

that the storm track length depends on the planetary rotation rate. For Earth's planetary rotation rate, the storm track length is similar to the width of the ocean basins, without continents being necessary for storm track termination. Using an idealized GCM with simple representations of zonal asymmetries allows us to isolate mechanisms controlling the dynamics of storm tracks, while being able to reproduce a mean climate that resembles Earth's. We show that the zonal asymmetries and the stationary eddies that they induce lead to an enhancement of the total meridional energy transport in the atmosphere. This enhanced energy transport is likely involved in the downstream self-destruction of storm tracks.

\section{Idealized GCM}

The idealized GCM is a three-dimensional model of a spherical aquaplanet and solves the primitive equations for an ideal-gas atmosphere. The GCM is based on that used by O'Gorman and Schneider (2008), which resembles that of Frierson et al. (2006). We use a horizontal spectral resolution of T85 and 30 vertical levels. For simulations with rotation rates $\Omega$ greater than $4 \Omega_{e}$, where $\Omega_{e}$ is Earth's rotation rate, we use the higher resolution of T127 to resolve the smaller energy-containing eddies. The lower boundary is a uniform slab ocean, with a heat capacity corresponding to $1 \mathrm{~m}$ of water. Radiative transfer is represented by a two-stream gray radiation scheme, and insolation is imposed as perpetual equinox. Moist convection is represented by a simplified Betts-Miller convection scheme [a variant of the scheme described in Frierson (2007)]. The scheme relaxes temperatures in convection toward a moist adiabat, and water vapor toward a profile with fixed relative humidity relative to the moist adiabat.

To generate storm tracks in the aquaplanet GCM, we introduce zonal asymmetries by imposing a localized ocean heat flux convergence in a triangular midlatitude region. This is an idealized representation of the area of warm ocean waters and strong atmospheric heating in the Gulf Stream or Kuroshio western boundary currents (Fig. 1c). The localized heating is applied between latitudes $25^{\circ}$ and $50^{\circ} \mathrm{N}$ (Fig. 2). The magnitude of the heat flux convergence is chosen such that the resulting enhanced atmospheric temperature gradients resemble those in Earth's storm track entry regions (Fig. 1a). However, our results do not depend qualitatively on the particular shape, size, or magnitude of the imposed heat flux convergence. Varying the heating amplitude modifies the storm track strength (EKE), but it does not substantially affect its length. In the Southern Hemisphere (SH), an ocean heat flux convergence is imposed of the same magnitude in the zonal mean as in the Northern Hemisphere but spread zonally symmetrically over all longitudes. This allows us to treat the Southern Hemisphere as a control case with the same zonal-mean thermal driving as the Northern Hemisphere but without a zonal asymmetry in boundary conditions. The results we present are averages from flow fields sampled 4 times a day for at least $2 \mathrm{yr}$, after a long spinup period in which the GCM statistics reached a steady state.

\section{Self-destruction of storm tracks}

On Earth, the Northern Hemisphere storm tracks occupy significant parts of the midlatitudes (Fig. 1b). Therefore, it is difficult to separate observationally the effects that storm tracks have on each other, continental effects, and self-maintained properties of each storm track. To show to what extent storm tracks can maintain themselves and to separate the scale of the storm tracks more clearly from the planetary scale, we conducted simulations in which we varied the planetary rotation rate.

A simulation with Earth's rotation rate already demonstrates that storm tracks self-destruct downstream (Fig. 2, left column). The localized heating creates a region of high EKE downstream (Fig. 2b), as a result of 


$$
\Omega=\Omega_{e}
$$

a



b

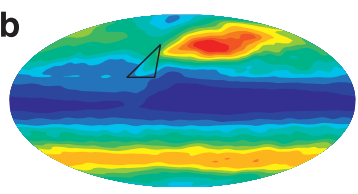

c

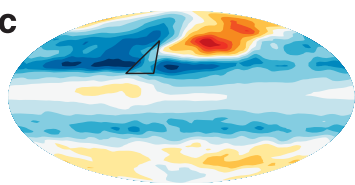

$\Omega=2 \Omega_{e}$
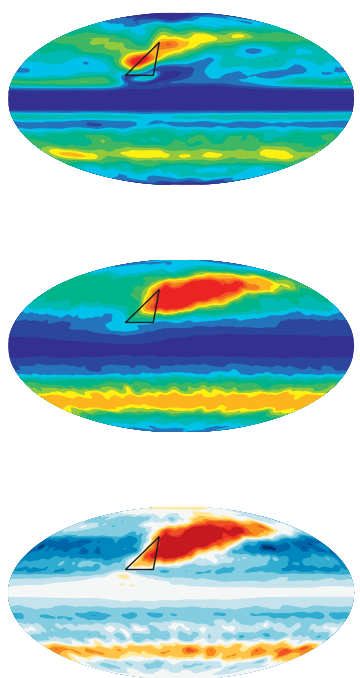

$\Omega=4 \Omega_{e}$
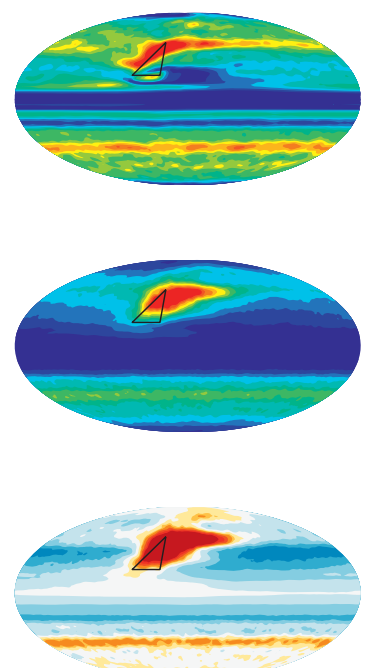
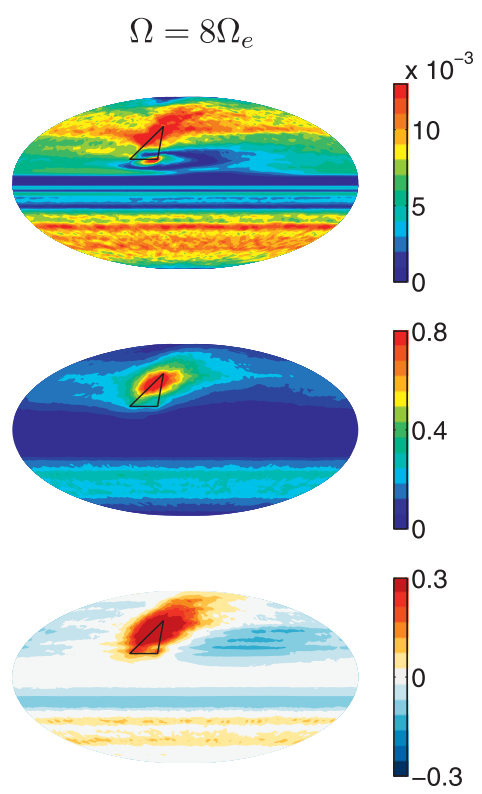

FIG. 2. (a) Absolute value of meridional temperature gradient averaged between $300 \mathrm{hPa}$ and the surface (K km ${ }^{-1}$ ). (b) Vertically integrated transient EKE $\left(\mathrm{MJ} \mathrm{m}^{-2}\right)$. (c) Transient EKE difference between simulations with and without the added ocean heat flux convergence in midlatitudes $\left(\mathrm{MJ} \mathrm{m}^{-2}\right)$. The EKE fields are vertically integrated. Results are shown for planetary rotation rates of $\Omega_{e}, 2 \Omega_{e}$, $4 \Omega_{e}$, and $8 \Omega_{e}$ (columns). As the rotation rate increases, the $\mathrm{NH}$ storm track becomes more confined longitudinally; EKE away from the storm track is generally smaller both than in the statistically zonally symmetric SH and than in simulations without added localized heating.

the increased baroclinicity (Fig. 2a) and direct diabatic generation of eddy energy over and downstream of the heating region. Although the model has no continents, the size and shape of the storm track resembles those on Earth (cf. Fig. 1b). Farther downstream, the EKE does not return to the level that would prevail in midlatitudes without zonal asymmetries in the heating or without any added midlatitude heating. Instead, the EKE settles to even lower values (cf. the Northern and Southern Hemispheres in Fig. 2b, and see Fig. 2c, which shows the difference in EKE between the simulation with the added heating in both hemispheres and a simulation without the added heating). Thus, the localized heating leads not only to an EKE increase in the storm track but also to EKE depression downstream. The region of depressed EKE persists not only immediately downstream of the storm track but also over the full latitude circle (Fig. 2c).

The downstream self-destruction of storm tracks occurs even as the planetary rotation rate is varied. As the rotation rate is increased, some important dynamical length scales such as the energy-containing transient eddy scale (e.g., Schneider and Walker 2006) and the stationary wave scale (e.g., Held et al. 2002) decrease. The storm track also shortens and becomes longitudinally and latitudinally more confined (Fig. 2b). For the high rotation rate cases, although there are multiple jets and baroclinic zones in each hemisphere, the storm tracks still exhibit one main EKE maximum. (However, in a more realistic model with a dynamical ocean and with multiple ocean gyres, the surface heating would change as well.) In addition, as in the $\Omega_{e}$ case, the EKE downstream is significantly lower both than in the Southern Hemisphere (without zonal asymmetries in heating) and than in a simulation without added heating (Fig. 2c). The downstream depression in EKE is almost uniform longitudinally, except in the simulations with rotation rates greater than or equivalent to $8 \Omega_{e}$, in which a partial recovery occurs far downstream.

The longitudinal EKE distribution is revealed more clearly in Fig. 3. It is evident that the EKE increases sharply in response to the localized heating in the Northern Hemisphere (red lines). Farther downstream, it consistently falls below the level it has with a corresponding zonally symmetric heating in the Southern Hemisphere (blue lines) or without any added heating (black lines). The existence of the longitudinally localized baroclinic zone causes a roughly $25 \%$ EKE depression downstream compared with a corresponding simulation without the added heating. The zonalmean EKE in the zonally asymmetrically heated Northern Hemisphere is also smaller than that in the zonally symmetrically heated Southern Hemisphere. Zonalmean EKE generally decreases with increasing planetary rotation rate because the smaller eddies can only convert 




FIG. 3. Midlatitude EKE (averaged between $30^{\circ}$ and $60^{\circ}$ latitude) as a function of longitude, for $\Omega_{e}, 2 \Omega_{e}, 4 \Omega_{e}$, and $8 \Omega_{e}$ : zonally asymmetrically heated NH (red), zonally symmetrically heated SH (blue), and simulations without added heating (black). Dashed vertical lines demarcate the longitudes of the added heating. The simulations correspond to those in Fig. 2. Downstream of the storm track, EKE is lower in the zonally asymmetrically heated cases than in other cases, demonstrating the self-destruction of the storm track.

the mean-flow potential energy in a meridionally narrower baroclinic zone to EKE (Schneider and Walker 2008). However, the relative EKE depression of $25 \%$ away from the heating region remains roughly constant as the rotation rate varies. The zonally symmetrically heated Southern Hemisphere (blue) has larger EKE than the corresponding unperturbed case (black) because the added heating enhances the baroclinicity.

Energy fluxes associated with storm track eddies are likely involved in the downstream self-destruction of the storm tracks. The zonal-mean poleward moist static energy flux $[\overline{v m}]$, averaged between $30^{\circ}$ and $60^{\circ}$ latitude, is enhanced in the Northern Hemisphere with the localized heating (Fig. 4a, red line) compared both with the statistically zonally symmetric Southern Hemisphere (blue line) and with the corresponding simulations without added heating (black line). \{Herein, [-] denotes the zonal mean, $\overline{(\cdot)}$ the time mean, $m=s+L q$ the moist static energy, and $s=c_{p} T+g z$ the dry static energy; otherwise the notation is standard (e.g., Peixoto and Oort 1992).

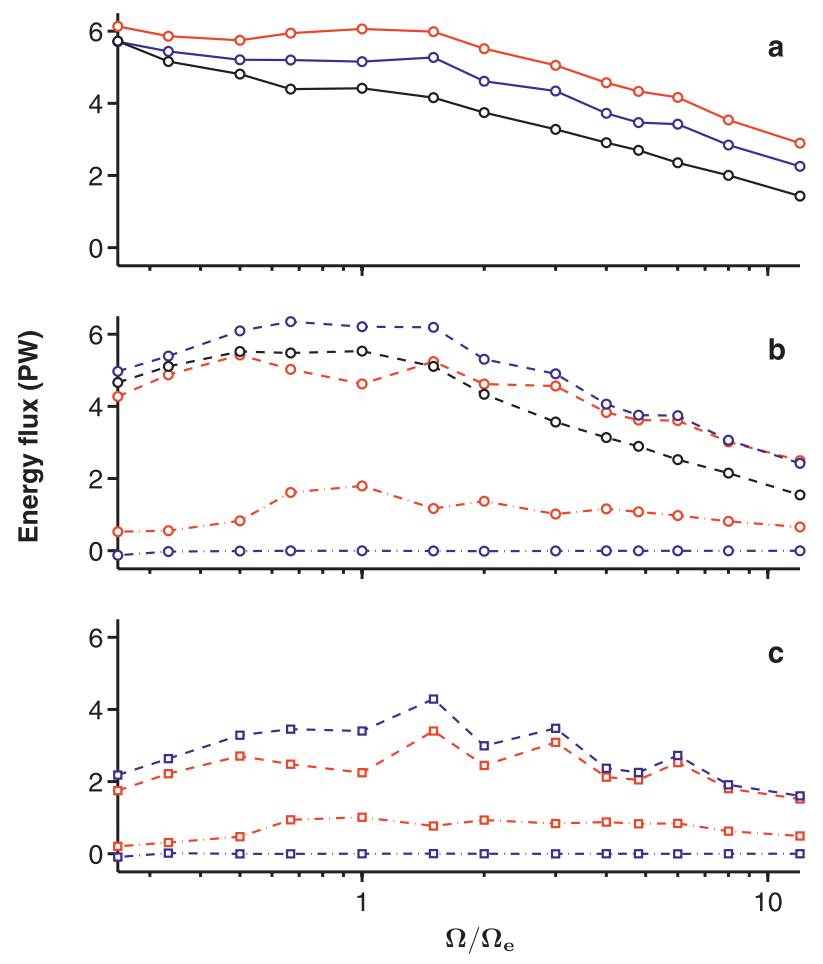

FIG. 4. Zonal-mean poleward energy fluxes as function of rotation rate $\left(\Omega / \Omega_{e}\right)$, averaged between $30^{\circ}$ and $60^{\circ}$ latitude. (a) Total flux $[\overline{v m}]$ for the $\mathrm{NH}$ with the added localized heating (red), for the SH with the added zonally symmetric heating (blue), and for the corresponding control simulations without added heating (black). (b) Transient eddy flux $\left[\overline{v^{\prime} m^{\prime}}\right]$ (dashed) and stationary eddy flux $\left[\bar{v}^{*} \bar{m}^{*}\right]$, (dashed-dotted) components for the same cases. (c) As in (b), but for the dry static energy $s$ flux only.

For all rotation rates, the moist static energy flux is enhanced by approximately 1 PW because of the existence of the zonal asymmetry. This enhanced poleward energy flux reduces meridional temperature gradients and thus baroclinicity in midlatitudes downstream of the localized heating and in the zonal mean.

The enhancement of the poleward moist static energy flux results because the stationary eddy fluxes $\left[\bar{v}^{*} \bar{m}^{*}\right]$, associated with the zonal asymmetry, overcompensate any reduction in transient eddy fluxes $\left[\overline{v^{\prime} m^{\prime}}\right]$ that arises because of the reduced zonal-mean baroclinicity (Fig. 4b). [Here, $(\cdot)^{\prime}$ denotes a fluctuation about the time mean and $(\cdot)^{*}$ a fluctuation about the zonal mean.] For the statistically zonally symmetric cases, the stationary eddy fluxes are by definition zero, and the transient eddy flux is somewhat stronger than the total moist static energy flux (cf. Fig. 4a) because of the contribution of thermally indirect mean meridional circulations (Ferrel cells). The transient eddy fluxes and total fluxes are stronger in the Southern Hemisphere with the added zonally symmetric heating compared with the corresponding simulations without 
added heating, as a result of the increased baroclinicity caused by the added heating. In contrast, the transient eddy fluxes in the Northern Hemisphere with the same zonal-mean heating but localized in longitude are always weaker in the zonal mean than those in the Southern Hemisphere (Fig. 4b).

Responsible for the enhanced total energy flux in the presence of zonal asymmetries are the stationary eddies (Fig. 4b) generated in response to the localized heating (e.g., Hoskins and Karoly 1981). Their overcompensation of any reduction in transient eddy fluxes appears to lead to a mean-flow modification downstream that is sufficiently strong to reduce EKE there below the values that would be obtained without zonal asymmetries. The total poleward moist static energy transport is about $20 \%$ stronger when the heating is longitudinally localized than when the same heating is spread uniformly around latitude bands. The dry static energy and latent heat $L q$ components contribute roughly equally to stationary and transient moist static energy fluxes (Figs. 4b,c); in the simulation with Earth's rotation rate, their magnitudes are similar to those in Earth's atmosphere (Trenberth and Caron 2001; Trenberth and Stepaniak 2003).

We hypothesize that the enhanced poleward energy flux reduces baroclinicity downstream and thus leads to the self-destruction of storm tracks. Baroclinicity is affected both by meridional temperature gradients and by the static stability. The poleward moist static energy flux reduces meridional temperature gradients and, as we will discuss elsewhere, enhances the static stability, particularly through the release of latent heat. The reduction of baroclinicity downstream causes the EKE depression and the termination of the storm track, overcompensating the enhanced baroclinicity in the heating region. The EKE depression persists downstream through advection by the zonal-mean flow $\bar{u}$. The fact that for the high rotation rate cases the storm track partially recovers far downstream (Fig. 3) shows that the depression is a downstream rather than an upstream effect, such as might be associated with an upstream Rossby wave plume (e.g., Rhines 2007; Kaspi and Schneider 2011).

Radiation can only gradually overcome the downstream depression and restore EKE to the values that would prevail without the zonal asymmetry on length scales of order $\bar{u} \tau$, where $\tau$ is a radiative time scale. For $\bar{u} \sim 10 \mathrm{~m} \mathrm{~s}^{-1}$ and $\tau \sim 30$ days, this gives a length scale of order $26 \times 10^{3} \mathrm{~km}$, or $330^{\circ}$ longitude at $45^{\circ}$ latitude, broadly consistent with the recovery scale seen in the rapidly rotating simulations (Fig. 3). This is an order of magnitude estimate, the precise value of the recovery length being uncertain, but it shows that the depression is expected to persist around a latitude circle for storm tracks of length similar to those on Earth.

\section{Conclusions}

Using a series of simulations with an idealized GCM, we have demonstrated that longitudinally localized heating leads to increased baroclinicity and the formation of storm tracks (local maxima in EKE) with well-defined length scales. Downstream of the storm tracks, EKE is depressed below the values that would be obtained without the added heating or with an equivalent longitudinally uniform heating. As the rotation rate of the planet increases, eddy length scales become smaller, and the storm track becomes more longitudinally confined. However, the EKE depression extends far downstream of the storm tracks. The EKE depression likely arises because stationary eddies associated with the localized storm track lead to an enhancement of the poleward moist static energy transport beyond that which would be obtained without the added heating or with an equivalent longitudinally uniform heating. This reduces baroclinicity downstream and thus depresses EKE.

On Earth, EKE is depressed over the continents downstream of both the Pacific and Atlantic storm tracks (Fig. 1b). It has been suggested that the increased mechanical eddy damping over land reduces the EKE (e.g., Chang and Orlanski 1993; Mak and Deng 2007), and GCMs with continents show that this is a possibility (e.g., Chang 2009). However, our simulations show that even without any horizontally varying damping, storm tracks terminate over roughly the same length scale as seen in Earth's atmosphere. Storm tracks have an intrinsic length scale apparently controlled by the eddies associated with them, which lead to their downstream self-destruction. We will discuss the mechanisms controlling this length scale in a forthcoming paper.

Acknowledgments. We thank Isaac Held, Xavier Levine, and Tim Merlis for useful discussions and comments. This research was supported by the NOAA Climate and Global Change Postdoctoral Fellowship administered by the University Corporation for Atmospheric Research, by a David and Lucile Packard Fellowship, and by NSF Grant AGS-1019211. The GCM is based on the Flexible Modeling System of the Geophysical Fluid Dynamics Laboratory; the simulations were performed on Caltech's Division of Geological and Planetary Sciences Dell cluster.

\section{REFERENCES}

Blackmon, M. L., 1976: A climatological spectral study of the 500 $\mathrm{mb}$ geopotential height of the Northern Hemisphere. J. Atmos. Sci., 33, 1607-1623.

, J. M. Wallace, N.-C. Lau, and S. L. Mullen, 1977: An observational study of the Northern Hemisphere wintertime circulation. J. Atmos. Sci., 34, 1040-1053. 
Broccoli, A. J., and S. Manabe, 1992: The effects of orography on midlatitude Northern Hemisphere dry climates. J. Climate, $\mathbf{5}$, $1181-1201$.

Chang, E. K. M., 1993: Downstream development of baroclinic waves as inferred from regression analysis. J. Atmos. Sci., 50, 2038-2053.

— 2009: Diabatic and orographic forcing of northern winter stationary waves and storm tracks. J. Climate, 22, 670-688.

— , and I. Orlanski, 1993: On the dynamics of a storm track. J. Atmos. Sci., 50, 999-1015.

— - S. Lee, and K. L. Swanson, 2002: Storm track dynamics. J. Climate, 15, 2163-2183.

Frierson, D. M. W., 2007: The dynamics of idealized convection schemes and their effect on the zonally averaged tropical circulation. J. Atmos. Sci., 64, 1959-1976.

—, I. M. Held, and P. Zurita-Gotor, 2006: A gray-radiation aquaplanet moist GCM. Part I: Static stability and eddy scale. J. Atmos. Sci., 63, 2548-2566.

Frisius, T., F. Lunkeit, K. Fraedrich, and I. N. James, 1998: Stormtrack organization and variability in a simplified atmospheric global circulation model. Quart. J. Roy. Meteor. Soc., 124, 1019-1043.

Held, I. M., M. Ting, and H. Wang, 2002: Northern winter stationary waves: Theory and modeling. J. Climate, 15, 2125-2144.

Hoskins, B. J., and D. J. Karoly, 1981: The steady linear response of a spherical atmosphere to thermal and orographic forcing. J. Atmos. Sci., 38, 1179-1196.

— J. Atmos. Sci., 47, 1854-1864.

Kaspi, Y., and T. Schneider, 2011: Winter cold of eastern continental boundaries induced by warm ocean waters. Nature, 471, 621-624.

Lau, N.-C., 1978: On the three-dimensional structure of the observed transient eddy statistics of the Northern Hemisphere wintertime circulation. J. Atmos. Sci., 35, 1900-1923.

, 1979: The structure and energetics of transient disturbances in the Northern Hemisphere wintertime circulation. J. Atmos. Sci., 36, 982-995.

Lee, S., 1995: Localized storm tracks in the absence of local instability. J. Atmos. Sci., 52, 977-989.
_ and I. M. Held, 1993: Baroclinic wave packets in models and observations. J. Atmos. Sci., 50, 1413-1428.

Mak, M., and Y. Deng, 2007: Diagnostic and dynamical analyses of two outstanding aspects of storm tracks. Dyn. Atmos. Oceans, 43, 80-99.

O'Gorman, P. A., and T. Schneider, 2008: The hydrological cycle over a wide range of climates simulated with an idealized GCM. J. Climate, 21, 3815-3832.

Orlanski, I., 1998: Poleward deflection of storm tracks. J. Atmos. Sci., 55, 2577-2602.

_ Southern Hemisphere. Part I: Eddy energy budget. J. Atmos. Sci., 48, 1972-1998.

Peixoto, J. P., and A. H. Oort, 1992: Physics of Climate. American Institute of Physics, $520 \mathrm{pp}$.

Rhines, P. B., 2007: Jets and orography: Idealized experiments with tip jets and Lighthill blocking. J. Atmos. Sci., 64, 3627-3639.

Schneider, T., and C. C. Walker, 2006: Self-organization of atmospheric macroturbulence into critical states of weak nonlinear eddy-eddy interactions. J. Atmos. Sci., 63, 1569-1586.

$\longrightarrow$, and — 2008: Scaling laws and regime transitions of macroturbulence in dry atmospheres. J. Atmos. Sci., 65, 2153-2173.

Simmons, A. J., and B. J. Hoskins, 1979: The downstream and upstream development of unstable baroclinic waves. J. Atmos. Sci., 36, 1239-1254.

— , and - 1980: Barotropic influences on the growth and decay of nonlinear baroclinic waves. J. Atmos. Sci., 37, 16791684.

Son, S., M. Ting, and L. M. Polvani, 2009: The effect of topography on storm-track intensity in a relatively simple general circulation model. J. Atmos. Sci., 66, 393-411.

Swanson, K. L., P. J. Kushner, and I. M. Held, 1997: Dynamics of barotropic storm tracks. J. Atmos. Sci., 54, 791-810.

Trenberth, K. E., and J. M. Caron, 2001: Estimates of meridional atmosphere and ocean heat transports. J. Climate, 14, 3433 3443.

— , and D. P. Stepaniak, 2003: Covariability of components of poleward atmospheric energy transports on seasonal and interannual timescales. J. Climate, 16, 3691-3705. 